\title{
Update in Pediatric Neurotrauma
}

\author{
Sarah Murphy ${ }^{1} \cdot$ Ann-Christine Duhaime ${ }^{1}$
}

Published online: 7 November 2016

(C) Springer International Publishing AG 2016

\begin{abstract}
Purpose of Review Globally, the incidence of traumatic brain injury (TBI) is increasing with significant costs and consequences to society.

Recent Findings Despite a tremendous research effort, however, there have been few clear, specific advances in the care of patients with severe TBI over the last 25 years. Multicenter randomized controlled trials (RCTs) have been conducted to investigate promising potential neuroprotective therapies in TBI, notably for hypothermia and progesterone among others, but none have demonstrated a clear therapeutic benefit. Because of the heterogeneity in age, injury type, and other factors, large-scale, appropriately powered studies are notoriously difficult to undertake in pediatric head injury. There remain few high-level evidence-based treatment recommendations for children with TBI.

Summary The increased use of MRI and other advanced imaging modalities may help direct pathoanatomic classification and individualized treatment of head injury in children.
\end{abstract}

Keywords Traumatic brain injury $\cdot$ TBI $\cdot$ Pediatrics $\cdot$ Injured child $\cdot$ Head injury $\cdot$ Neurotrauma

This article is part of the Topical Collection on Pediatric Trauma

Sarah Murphy

SAMURPHY@mgh.harvard.edu

Ann-Christine Duhaime

aduhaime@partners.org

1 Wang Ambulatory Care Center, Suite 331, Neurosurgical Service, Massachusetts General Hospital, Fruit Street, Boston, MA 02114, USA

\section{Introduction}

Traumatic brain injury (TBI) has been called "the most complex disease in the most complex organ" [1] and is characterized by tremendous heterogeneity in mechanism, pathology, and severity. TBI is a leading cause of death and life-long disability in children [2-5]. Data from the CDC indicate that more than 1.7 million people and more than 500,000 children sustain a TBI in the USA each year. More than 2000 US children die from their acute TBI and many more will survive with life-long repercussions $[3,6]$. The CDC estimates that 145,000 children and adolescents are living with substantial limitations in social, behavioral, physical, or cognitive functioning following a TBI [7]. These numbers may underestimate the true burden of injury, given the likely under-reporting of mild TBI (mTBI), concussion, and abusive head trauma [8].

The potential physical, cognitive, and behavioral sequela of TBI include neurologic complications such as motor weakness and spasticity, epilepsy, hydrocephalus, headache syndromes, and autonomic disturbances, as well as cognitive impairments such as deficits or impairments in attention, memory, executive function, and processing speeds $[9 \bullet, 10,11 \bullet]$. The impact of TBI on a child's social development is less well understood but may be one of the most disabling and longlasting consequences of injury [12]. Investigators have linked pediatric TBI to aggressive and maladaptive behavior and poor social adjustment and engagement. A longitudinal study of pediatric patients showed that recovery profiles differed as a function of injury severity. Children with severe TBI had significant social problems that were continuing to evolve between 12 and 24 months post-injury when compared with uninjured controls and children with only mild or moderate TBI. In contrast, children with mild and moderate injuries showed only few social problems at 12 months post-injury and little change over time [12]. It has been hypothesized that 
the susceptibility of the frontotemporal lobes to injury, situated above boney surfaces where brain tissue can be easily impacted by force, may be responsible for the cognitive and behavioral symptoms commonly seen after a TBI [13] (CDC report 2015). How TBI disrupts developing, widespread neural networks in pediatric patients is poorly understood, a subject of intense research, and undoubtedly consequential [11•].

Worldwide, there is a global pandemic of TBI, with significant societal cost and consequence [14••]. The incidence of TBI in developing countries is increasing, particularly in middle and low-income countries with increasing use of motor vehicles, and TBI is expected to surpass many other diseases as a major cause of death and disability by 2020 according to the World Health Organization $[15,16]$. In the USA, the Nationwide Emergency Department Sample database (NEDS), representing a patient sample from 950 participating hospitals, suggests that emergency department (ED) visits for TBI have increased $29.1 \%$ between 2006 and 2010, which is $8 \times$ the percent increase in total ED visits [17]. The majority of these are coded as concussion or "head injury other." Patients at the extremes of age, adults $>60$ years and children $<3$ years, had the greatest increase in injuries over this time period, perhaps reflecting that these populations are least impacted by public health efforts such as traffic safety laws and concussion prevention measures [17].

More than half of the TBI among children 0 to 14 years was caused by falls (55\%) (CDC website). Unintentional blunt trauma (e.g., being hit by an object) accounted for close to a quarter $(24 \%)$ of all TBIs in children, and motor vehicle crashes were the third overall leading cause of TBI (14\%) but are responsible for a disproportionate portion of mortality. Motor vehicle crashes were the leading cause of TBI-related death for children and young adults aged 5-24 years while assault is the leading cause of TBI-related death for children aged $0-4$ years. With over 7000 pediatric deaths reported in the USA in 2005, TBI is a leading killer of children in the USA and is estimated to cost more than $\$ 2$ billion dollars each year for acute care alone. The societal costs, including lifetime costs of disability, lost life years, and lost productivity, are high $[14 \bullet \bullet, 18,19]$.

Despite a tremendous research effort, there have been few clear, specific advances in the care of patients with severe TBI over the last 25 years. Multiple multicenter randomized controlled trials (RCTs) have been conducted to investigate promising potential neuroprotective therapies in TBI, but none have demonstrated a clear therapeutic benefit, and some metaanalyses have shown that improvements in mortality and morbidity rates for people with severe TBI have stalled [20]. Neuroprotective therapies that have been investigated over the last 25 years include therapeutic hypothermia, progesterone, decompressive craniectomy, and intracranial pressure (ICP) monitoring. Of these, only hypothermia has been studied in an RCT in a pediatric population. Though the initial trial of therapeutic hypothermia to $33{ }^{\circ} \mathrm{C}$ for $48 \mathrm{~h}$ in adults with severe TBI (NABISH) [21] did not demonstrate a benefit in terms of mortality or outcome, subgroup analysis suggested the possibility of a modest benefit in a subgroup of younger TBI patients $(<45$ years). This effect, however, was not borne out in the follow-up study by the same investigators [22] targeting a younger adult population $(<45$ years) with earlier cooling. In subsequent years, the Canadian Critical Care Trials Group study "Hypothermia after TBI in children" [23•] and the multinational CoolKids study [24•] both failed to find a benefit to cooling to $32-33^{\circ} \mathrm{C}$ for 24 or $48-72 \mathrm{~h}$, respectively, in pediatric patients with severe TBI. In the Hutchison trial, children treated with hypothermia had a trend toward higher mortality and an increased risk of developing hypotension during rewarming. The CoolKids trial was terminated for futility after interim analysis failed to demonstrate a benefit. In a detailed feasibility analysis conducted in a third study of hypothermia following pediatric sTBI (phase II pilot), Beca et al. noted that given the low randomization rates in pediatric hypothermia trials (ranging from 4.2 to $15 \%$ ), between 3000 and 10,000 patients would need to be screened in order to enroll enough patients to detect a $10 \%$ absolute reduction in poor outcome [25], making the completion of such a trial unfeasible.

Over the same period, multicenter RCTs in adults have failed to find clinical crossover of the preclinical benefits seen from progesterone therapy in TBI $[26,27]$ or to find evidence of efficacy from bifrontal cranial decompression (discussed in detail below). The routine use of ICP monitoring, a fundamental tenet of acute care in severe TBI management for nearly 30 years, was found to provide no outcome or mortality benefit in a recent international RCT conducted in Ecuador and Bolivia compared to careful serial examination and serial imaging. In this study involving 324 patients aged 13 years or older, guideline-based management, guided by ICP-monitoring, did not result in better outcomes than those that could be achieved through aggressive treatment based on clinical examination and head imaging alone [28•].

In 1995, the Brain Trauma Foundation first published guidelines for the care of adults with severe TBI that included a recommendation for intracranial pressure monitoring and stepwise treatment of ICP above a threshold of 20 $25 \mathrm{mmHg}$. Pediatric guidelines followed in 2003 and were most recently revised in 2012; these had disappointingly little new grade I or II evidence to incorporate into best practice. In fact, best practices for most aspects of TBI care in children have not been established [14••, 29]. The most recent pediatric guidelines include no level I and only a handful of level II recommendations, including recommendations about hypothermia that are at variance with the results of the most recent trial results) [4]. Other grade II recommendations include lack of evidence for an immune-modulating diet to improve outcome and lack of benefit of corticosteroids to improve 
outcomes or reduce edema. The remaining recommendations, including those regarding ICP monitoring and treatment, minimum cerebral perfusion pressure (CPP) goals, use of analgesics and sedatives, and others, are level III recommendationexpert opinions [4] .

Because of the heterogeneity in injury type, age, and other factors, large-scale, appropriately powered studies on which to base high-level-evidence standards are notoriously difficult to undertake in pediatric head injury, and for this reason, much treatment is individualized. This is particularly true with increased use of MRI and other imaging modalities that can help with pathoanatomic classification rather than treatments based solely on Glasgow Coma Scale (GCS) [5]. In light of the fact that guidelines documents contain many options based on weak evidence and few high-quality evidence-based standards, it may not be surprising that the extent to which centers adhere to currently published guidelines documents is variable [30-32]. Additionally, how "compliance" is measured in studies of this type is variable, since many of the recommendations are only at the "option" level, making interpretation of adherence problematic. In a recent US survey of 245 adult trauma center directors, $83.3 \%$ reported that the guidelines were completely followed at their center in less than $75 \%$ of severe TBI cases and $32.2 \%$ reported that the guidelines are followed in less than $50 \%$ of adult patients presenting with severe TBI [31]. This is consistent with previous reports of large variance in percent compliance with guidelines within and across centers. As in other studies [32, 33], ICP monitoring and also the use of hypertonic saline were the most contentious recommendations with the lowest rate of reported compliance. A majority of directors $(61.2 \%)$ stated that ICP policies were implemented in less than $60 \%$ of sTBI cases, and $60.1 \%$ of directors reported the hypertonic saline is used in compliance with the guideline recommendations less than $60 \%$ of the time [31]. To improve clinical practice and consistency in care in TBI, there has been an emphasis on improving guideline adherence in adult trauma patients in the USA and a trend toward better overall compliance since their inception in 1995 [34]. But, the evidence regarding whether consistency with the guidelines actually improves outcomes is mixed. Though some single-center studies have suggested improvement in mortality and outcome with higher guideline adherence [32, $35,36]$, more recent investigations had found little or no association between complete/high levels of guideline compliance [33] or compliance with specific recommended measures (ICP monitoring, craniotomy) and risk-adjusted outcomes of adult patients with severe TBI $[33,37]$. However, there is a well-documented "halo" effect seen as improved outcomes in both treatment and control groups in clinical trials (mostly in adults), suggesting that attention to detail and protocol-driven management may have major benefits [1].

Despite the lack of supporting evidence, the idea of having pediatric guidelines available, with review of currently available evidence, has been generally well received [38]. As might be expected from the variable strength of evidence, adherence to published recommendations is variable both between and within centers. This has been most well studied in regard to the issue of ICP monitoring. In a recent US National Trauma Database review of pediatric patients with severe TBI from 2001 to 2006, only $7.7 \%$ of patients meeting the criteria underwent ICP monitoring. Notably, ICP monitoring in this study was associated with decreased mortality only when the GCS was 3 but was associated with longer ICU stay, longer hospitalization, and more days on the ventilator, potentially reflecting the population most likely to be chosen to undergo monitoring [39]. In the past decade, reported rates of ICP monitoring in the pediatric population have ranged from 7.7 to $59 \%$ [39-43]. It has been shown that infants less than 1 year are less likely to undergo ICP monitoring [41, 42] than are older children. Additionally, children hospitalized at adult-only trauma centers are more likely to have ICP monitors placed than are pediatric patients cared for at pediatric trauma centers [41]. The reasons for variable compliance with guideline recommendations likely include, in addition to factors related to implementation, dissemination, culture and communication, meaningful questions about guideline credibility, and the applicability of specific recommendations for an individual patient [42, 44]. In one study, rapid early improvement in GCS, decision to pursue clinical surveillance, and moribund status, for example, were the most common reasons found for why ICP monitoring was not implemented. In addition, in this study, radiologic (CT or MRI) features were also noted to be an important factor in the decision whether or not to place an ICP monitor - a factor not included in the current guidelines. All of the patients who underwent clinical surveillance in this study had Rotterdam CT scores of 1, 2, or 3 (most often 1-2) [42].

Vavilala et al. studied attainment of 34 clinical care indicators derived from the 2003 guideline recommendations in 5 high-volume level I pediatric trauma centers through a retrospective review of cases from 2007 to 2011 [45]. Overall compliance with the clinical indicators defined by the study was $73 \%$ and ranged from 68 to $78 \%$, comparable to Brain Trauma Foundation guideline compliance rates reported in adults. A $6 \%$ lower hazard of death and a $1 \%$ decrease in the chance of poor (vegetative or major impairment) functional outcome was found for every percentage point increase in adherence with the indicators. After controlling for confounders, mortality and outcome were found to be associated each with three different clinical indicators. The indicators associated with reduced mortality included absence (but not treatment) of prehospital hypoxia, early ICU nutrition $(<72 \mathrm{~h})$, and no hyperventilation in the absence of evidence of herniation. The specific indicators associated with improved outcome in survivors were a CPP maintained $>40 \mathrm{mmHg}$ in the OR and ICU, respectively, and absence of a surgical intervention. This study provides indirect support of the goals of care 
put forth in head injury management recommendations, finding an association between related clinical indicators and outcome. However, the argument can be made that this reflects that patients in whom parameters cannot be controlled because of severe injury burden, such as patients in whom ICP or CPP cannot be maintained in a "target range," simply have worse outcomes on that basis, rather than providing evidence that specific interventions alter outcome. Single-center studies have demonstrated improvement in outcome after the adoption of local care processes consistent with guideline recommendations [46, 47]. Perhaps the most notable direct evidence for a pediatric guideline recommendation was provided in a study published in Pediatrics in 2009 [48]. In this retrospective study of pediatric patients presenting to a level I trauma center ED with severe TBI, hypoxia (44\%) and hypotension (39\%) were detected frequently in the ED. Non-treatment of hypotension (but not of hypoxia) was associated with a 3.4 increased odds of death and a 3.7 increased odds of disability. This is consistent with general principles of maintenance of physiologic stability and avoidance of so-called secondary insults to add to the injury burden.

There persist wide discrepancies in outcome even between "experienced" TBI centers that may impede the accurate assessment of neuroprotective therapies and strategies in multicenter, randomized controlled trials [49]. The adult International Mission on Prognosis and Clinical Trial design in Traumatic Brain Injury (IMPACT) trial analyzed individual patient data from over 9500 adult patients with moderate and severe traumatic brain injury enrolled in 10 randomized controlled trials and 3 observational studies [50 0 . A randomeffects logistic regression model was used to estimate the between-center differences in unfavorable outcome (dead, vegetative state, or severe disability measured with the Glasgow Outcome Scale) at 6 months adjusted for differences in patient characteristics. A 3.3-fold difference in the odds of having an unfavorable outcome was found between centers, a difference which was not explained by random variation or patient characteristics [50॰]. In fact, adjusting for patient characteristics increased between center differences. In this study, as in others, outcomes adjusted for patient characteristics did not improve over the study period (1984-2004), during which time guidelines were disseminated.

There is significant variability in pediatric TBI-related outcomes by state. A retrospective cohort study using Healthcare Cost and Utilization Project data found nearly a 2-fold difference between states in inpatient mortality after pediatric TBI [51]. Regional differences have also been shown in the outcome of patients with severe TBI even after adjusting for known facility and patient variables including trauma level, facility size and teaching status, age of patient, patient insurance status, measures of TBI severity, presence of hypotension, heart rate, age, transfer status, GCS, AIS, and other factors $[51,52]$. These results are in agreement with CDC estimates of TBI-related mortality in children, which have been lowest in the northeast (2.4 per 100,000), compared with the west (3.2 per 100,000), Midwest (3.9 per 100,000), and south (4.3 per 100,000) (CDC website). Disparities in outcomes have also been shown with minority and impoverished children having a higher incidence of TBI, worse severity of injury, and higher mortality rates related to their TBI $[53,54]$.

Such differences in outcome might be explained by differences in data collection and reporting, process and trauma system factors, differences in injuries or in the population, or differences in treatment and practice [52]. In 2013, Bell et al. published results of a survey that asked participants from 32 pediatric TBI centers from across the USA and Europe about their treatment goals for pediatric patients with severe TBI [55]. There were wide differences reported in stated medical goals, including differences in intracranial hypertension management; use of cerebrospinal fluid diversion; use of multimodal, brain tissue oxygenation monitoring; and initiation of nutritional support, among other areas.

For all these reasons, there remain many questions about current best practice in the management of sTBI in children. The current state of knowledge and the somewhat controversial results of recent trials including BEST-TRIP and others $[28 \cdot, 56,57]$ challenge some of the historic management recommendations for adults and children with sTBI. The next evolution in TBI care may be toward adding more sophisticated management algorithms into the care of patients, based on individual patients' unique pathophysiology of injury. This care might be guided by the use of advanced imaging and monitoring techniques that might allow targeted treatment and individualized treatment thresholds [29].

Recent focus in clinical TBI research has turned to leveraging the differences in care practices and medical treatment goals that currently exist. An international initiative including the National Institutes of Health (NIH) and the European Commission (EC) has sought to establish collaboration in TBI research by establishing common shared definitions and standards such as those described by the common data elements (CDE) for TBI. By using agreed-upon definitions and standards, large-scale shared databases of TBI patients can be created and studied. Through this collaboration, comparative effectiveness trials including Approaches and Decisions in Acute Pediatric TBI (ADAPT), Transforming Research and Clinical Knowledge in Traumatic Brain Injury (TRACKTBI), and the Collaborative European NeuroTrauma Effectiveness Research in Traumatic Brain Injury (CENTER-TBI) studies all aim to compare the relative benefits and harms of alternative treatments and strategies currently used in the care of patients with severe TBI through prospective data collection of highly granular clinical, neuroimaging, biomarker, and outcome data [14••]. ADAPT aims to evaluate TBI therapies specifically in children with severe TBI, including the use of intracranial hypertension strategies, 
use of prophylactic hyperventilation and brain tissue oxygen monitoring, and nutritional support.

\section{Surgical Management}

\section{General Principles}

The main goal of surgery is to relieve dangerous tissue shifts and, less commonly, to decrease globally increased intracranial pressure. Most instances of potentially preventable death and disability from traumatic brain injury in the hospital setting occur as a result of tissue shifts which cause progressive compromise of otherwise relatively spared areas of the brain. These areas can become secondarily ischemic, resulting in swelling and extension of the volume of compromised tissue. This can occur before intracranial pressure causes failure of global brain blood flow. Thus, prompt removal of mass lesions and creating "room" for contused tissue to swell away from undamaged tissue are the most common scenarios requiring surgical intervention.

\section{Extraaxial and Intraparenchymal Hematomas}

Patients presenting with extraaxial hemorrhages - epidural and subdural hematomas - are evaluated via the initial assessment algorithm (Fig. 1). It should be kept in mind that epidural hematomas in infants and young children most often occur from low-height falls, and that they may progress rapidly or, in the case of venous hemorrhages, more slowly [59]. Mass lesions causing immediate or potential compromise are best evacuated before irreversible injury occurs to adjacent tissue [59]. Mass lesions close to sites in which tissue shifts are more likely to cause compromise of vessels or of critical tissuesuch as those in the temporal region near the tentorial incisura and brainstem, those near the falx which can cause subfalcine herniation and compromise of the anterior circulation, and those in the posterior fossa which can cause brainstem compression or compromise of the posterior arterial circulation via herniation through the foramen magnum - are all managed with a high index of suspicion for potential deterioration. Additionally, extraaxial lesions which are not causing immediate compromise but are associated with contused parenchyma that can be predicted to swell as the injury evolves also decrease the surgeon's threshold for operative intervention. These lesion scenarios may prompt prophylactic surgical intervention to decrease the chance of sudden irreversible decline even before herniation is imminent.

Conversely, some mass lesions that do not compromise uninjured tissue and that can be predicted to have a high likelihood of stability can be treated conservatively initially but always with close observation in case unexpected worsening, clinically or radiographically, occurs. This is why the timing of the scan relative to the injury event can be so useful; a small, stable venous epidural hematoma associated with a simple fracture located far from structures associated with herniation in a well-appearing child may be well tolerated and may be managed non-operatively initially. Subdural bleeding in the setting of preexisting encephalomalacia falls into a similar category of lesion which has a lower risk of causing dangerous tissue shifts; this situation is more common in adults than in children. In borderline cases, such as a moderate epidural hematoma with persistent headache and vomiting but without major herniation risk, it is worth keeping in mind that symptomatic patients typically recover more quickly and leave the hospital sooner if surgery is performed early, rather than having patients undergo many days of observation and repeated scans, especially if imaging is performed with CT and its attendant radiation exposure [60]. Follow-up imaging of small, asymptomatic or minimally symptomatic epidural collections, especially if the first scan was obtained within hours of injury, is another context in which rapid MRI may have utility compared to CT.

Other mass lesions follow similar principles but can require more judgment. Intraparenchymal hemorrhages with mass effect also can compromise adjacent tissue, but surgery carries the additional risk of compromise of the surrounding brain which may have the potential to recover. This is particularly true in eloquent cortex, such as the temporal and frontal lobes, common locations of traumatic contusions which may include significant solid hemorrhage. While frontal contusions have been thought to be amenable to surgical intervention with little obvious consequence in the past, more recent recognition of the role of the frontal lobes in decision-making and executive functions, reward processing, and social interaction has made preservation of these areas more compelling. This may be particularly true in light of the fact that deficits from injury in this area may not be apparent until later in maturation for many children. Thus, surgical decision-making of significant traumatic hemorrhages in this area involves weighing goals of short- and longterm risks against immediate benefits of reduction of intracranial pressure and melioration of local mass effect.

Similar decision-making occurs regarding traumatic hemorrhages in the temporal lobe. This is why knowing the child's handedness may be very useful early in the course of care. While younger children may exhibit language plasticity, older children may exhibit expressive or receptive language deficits after surgical evacuation of parenchymal hematomas in the dominant temporal lobe and perisylvian region. However, because the temporal lobe is close to the brainstem, mid- or posterior-temporal hemorrhages carry significant risk, and judicious surgical evacuation may be prudent.

Deep hemorrhages, such as those in the basal ganglia, internal capsule, and thalamus, occur most commonly in the setting of high-speed rotational events, such as trauma 
I. Basic minimum acute data

History

- Time of injury

- Mechanism

- Kinematics; energy, speed, height

- Struck head?

- Best exam at scene

- Loss of/level of consciousness

- GCS

- Asymmetry (pupils, motor)

- Moved legs?

- Exacerbators

- Apnea

- Shock

- Prolonged extraction

- Other injuries

- Resuscitation

Exam

- Via standardized tool (GCS, motor, pupils, other)

Imaging

- Time of imaging

- Is there a mass lesion causing symptoms or significant tissue shift now or with predictable worsening?

Swelling/deterioration-
prone injuries
Acute subdural hematoma
(not in atrophic brain)
Multifocal/large contusions
Temporal or posterior fossa lesions
High energy contact injury
Gunshot wound
+ Exacerbators

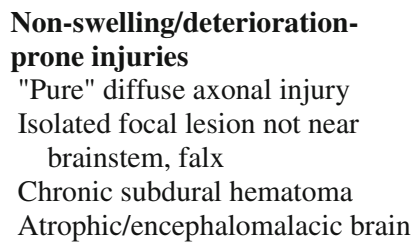

Fig. 1 Head injury: initial assessment and management for neurosurgical consultants. **Unoperated epidural hemtaoma, significant contusion, others at discretion of the treating physician. Adapted from [58]

including motor vehicles, often in concert with diffuse axonal injury. When life-threatening, these can be evacuated but carry risk of damage to surrounding tissue which requires some degree of disruption to remove the deep collection of blood. Careful planning of a trajectory to the hematoma that minimizes collateral risk is important, and the use of intraoperative ultrasound or image guidance, even under conditions of urgent surgery, can be extremely helpful in this circumstance.

\section{Contusions}

Mechanical trauma along brain surfaces can result in tissue contusions, most often in the frontal or temporal lobes, but these can occur in any area, including the posterior fossa. Contusions represent zones of damaged interspersed with potentially salvageable tissue, and in general, caution is used when evacuating these in order to preserve as much function as possible. However, as per the principles outlined above, when contused tissue swells to the point of progressive compromise of adjacent uninjured tissue or herniation, creating room for swelling via decompression and/or evacuation becomes important. It should be kept in mind that the majority of contusions swell over the first few days after injury, and minimizing this is the goal of many medical management of TBI.

\section{Intracranial Pressure Monitoring and Ventriculostomy}

The goal of intracranial pressure monitoring is to supplement the continuous evaluation of the head-injured patient beyond what serial neurologic exams and serial imaging can provide. In some instances, neither serial exams nor serial imaging can be performed or have high risk - for instance, when children have polytrauma requiring extracranial surgery, have painful injuries requiring sedating analgesics, have pulmonary function compromise requiring ventilation parameters only 
Is there a mass lesion causing symptoms or significant tissue shift now or with predictable worsening?

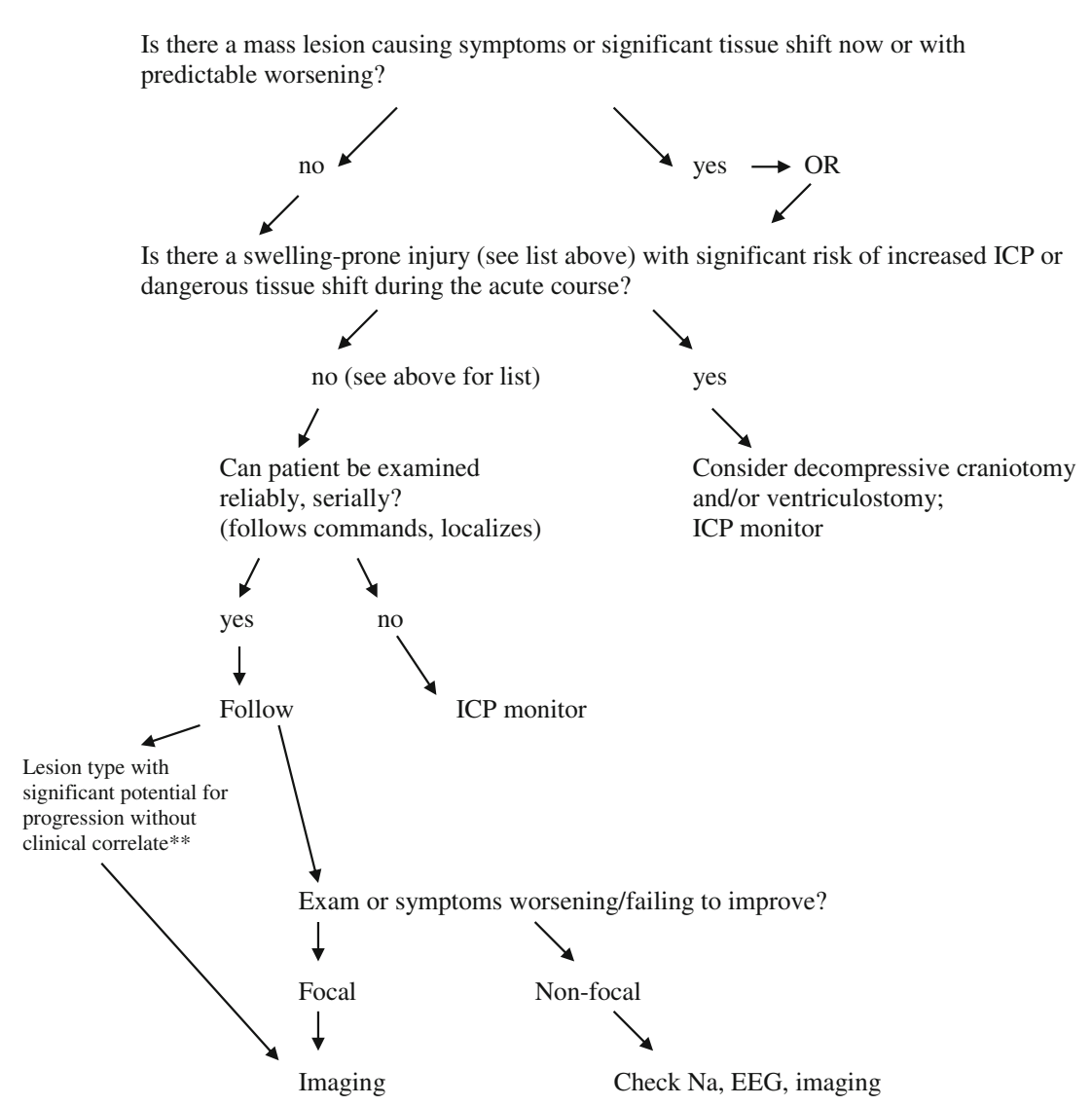

Fig. 1 continued.

possible with deep sedation or muscle paralysis, or have decreased level of consciousness related to the brain injury. While serial neurologic examination is a pillar of management and should always be the goal whenever possible, as it provides information about level of consciousness, new focal findings, pain management adequacy, as well as signs of recovery that can lead to de-escalation of care, intracranial pressure monitoring can provide supplementary information. In particular, trends to increases in intracranial pressure may be of great importance during many phases of management, including those mentioned above. While tissue shift and frank herniation can occur even without major increases in intracranial pressure, elevations can be a harbinger of delayed mass lesions, progressive swelling, ischemia, agitation, and other trends potentially requiring changes in management. Thus, intracranial pressure monitoring is generally instituted in all patients in whom it will add to the information available which may help prevent potentially avoidable deterioration. This includes children who are unconscious, who cannot undergo serial clinical examination with an interpretable exam (usually, one is which they can reliably follow command), or whose exam and findings are unreliable due to other needed medical interventions.

Ventriculostomy requires placement of a catheter into the ventricle and thus has a slightly higher risk of intraparenchymal hemorrhage as well as infection compared to intraparenchymal ICP monitors. However, ventriculostomy allows for continuous or intermittent drainage of CSF, which can be extremely useful in patients with brain swelling. Because children and adolescents often have little "extra" subarachnoid space compared to older adults, even relatively small volumes of swollen tissue can lead to dangerous tissue shifts and elevations of intracranial pressure. Thus, removal of even a few milliliters of CSF per hour in the setting in which the intracranial pressure can be elevated significantly by small increases in volume can add considerable safety to management. Thus, placement of ventriculostomy should be considered early in the setting of anticipated brain swelling that does not require more major forms of decompression, such as those discussed below. For lesions that do not fall into the "swelling-prone" category, such as 
isolated diffuse or traumatic axonal injury without contusion or superimposed hypoxic-ischemic injury, parenchymal ICP monitors offer a reliable method of monitoring changes in pressure, along with serial neurologic exams and serial imaging as clinically indicated. They also can be used to supplement ventriculostomy readings when continuous CSF drainage is chosen for management, to take advantage of their capacity for reliable, continuous ICP measurement; the authors typically use a fiberoptic parenchymal monitor in children requiring continuous ventriculostomy drainage as an extra means of following pressures. While these are general principles, the exact efficacy of these approaches in children awaits further prospective study [61].

\section{Brain Swelling and Decompressive Craniectomy}

While some contused tissue is best removed by surgical evacuation, other contusions can be handled by decompressive surgery, leaving the tissue largely intact. If an entire hemisphere or bifrontal region can be expected to swell, either because of prior evacuation of a subdural hematoma-a lesion often associated with hemispheric brain swelling - or because of large contusions, decompressive craniectomy can be performed. This has been employed with increasing frequency in the past decade and sometimes enables preservation of parenchyma during the period of maximal swelling while minimizing the risk of progressive compromise of adjacent tissue. Multiple studies have demonstrated that decompressive craniectomy, either bifrontal or, more often, unilateral, is associated with best outcomes if performed early in the course of predicted swelling [62-65]. Similar decompression can be accomplished in the setting of cerebellar contusions as well as large penetrating lesions such as some gunshot wounds in which significant tissue sparing may be anticipated. However, when physiologic damage results from hypoxic-ischemic injury or other diffuse metabolic insult, or when herniation infarcts have already occurred, decompression may be life-saving but with poor neurologic outcome [65]. Additionally, late complications, including shunt-dependent hydrocephalus and bone resorption, occur with high frequency after decompressive craniectomy. Thus, families need to be informed that multiple surgeries may be needed during what can be a long and intervention-laden recovery $[62,66]$.

Despite these cautions, decompressive craniectomy has come to be used more frequently in both adults and children and is considered a justified option in circumstances of swelling-prone lesions that can be anticipated to cause progressive compromise of potentially salvageable tissue that might be avoided by this surgical maneuver [67]

\section{Imaging}

For pediatric patients, there is significant concern about the long-term effects of radiation exposure-both increased risk of malignancy and the potential for cognitive injury have been reported from CT scans in children $\left[68^{\bullet}\right.$, 69-71]. For initial imaging, reduced radiation head and extracranial CT protocols exist, and these typically are sufficient for initial assessment and triage. For further assessment of significant brain or spine injuries, MRI has more sensitivity and specificity for parenchymal assessment and can often give additional information about acute injury as well as evolving pathophysiology of injury that might be used to direct further management. New, rapid MRI sequences can often be performed safely, limiting the amount of time that the patient is in the imaging suite. Some newer sequences, such as half-Fourier acquisition single-shot turbo spin-echo (HASTE), can scan the entire brain in $30 \mathrm{~s}$ and have the added advantage of being relatively resistant to motion artifact. MRI is used increasingly both in the acute setting (emergency department) and as the follow-up imaging modality of choice in pediatrics [72]. The National Institutes of Health, along with other government agencies, sponsored the creation of common data elements for traumatic brain injury, with CT and MRI parameters for children, which can be used to guide imaging decisions, protocols, and interpretation $[73,74]$.

\section{Conclusions}

There remain few high-level, evidence-based recommendations for the acute care of children with severe TBI. The increased use of advanced MRI imaging modalities may allow for more tailored, pathoanatomic management of head injury in children. Large-scale databases of TBI patients promise to advance our care of patients with severe TBI. Comparative effectiveness trials include Approaches and Decisions in Acute Pediatric TBI (ADAPT), Transforming Research and Clinical Knowledge in Traumatic Brain Injury (TRACKTBI), and the Collaborative European Neurotrauma Effectiveness Research in Traumatic Brain Injury (CENTER-TBI) and will compare the relative benefits and harms of alternative treatments and strategies currently used.

\section{Compliance with Ethical Standard}

Conflict of Interest Drs. Duhaime and Murphy declare no conflicts of interest relevant to this manuscript.

Human and Animal Rights and Informed Consent This article does not contain any studies with human or animal subjects performed by any of the authors. 


\section{References}

Papers of particular interest, published recently, have been highlighted as:

- Of importance

- Of major importance

1. Wheble JLC, Menon DK. TBI-the most complex disease in the most complex organ: the CENTER-TBI trial-a commentary. J R Army Med Corps. 2016;162:87-9.

2. Schneier AJ, Shields BJ, Hostetler SG, Xiang H, Smith GA. Incidence of pediatric traumatic brain injury and associated hospital resource utilization in the United States. Pediatrics. 2006;118:48392.

3. Langlois JA, Rutland-Brown W, Thomas KE. The incidence of traumatic brain injury among children in the United States: differences by race. J Head Trauma Rehabil. 2005;20:229-38.

4. Keenan HT, Bratton SL. Epidemiology and outcomes of pediatric traumatic brain injury. Dev Neurosci. 2006;28:256-63.

5. Faul M, Coronado V. Epidemiology of traumatic brain injury. Handb Clin Neurol. 2015;127:3-13.

6. Tagliaferri F, Compagnone C, Korsic M, Servadei F, Kraus J. A systematic review of brain injury epidemiology in Europe. Acta Neurochir (Wien). 2006;148:255-68. discussion 268.

7. Zaloshnja E, Miller T, Langlois JA, Selassie AW. Prevalence of long-term disability from traumatic brain injury in the civilian population of the United States, 2005. J Head Trauma Rehabil. 2008;23:394-400.

8. Leibson CL et al. Incidence of traumatic brain injury across the full disease spectrum: a population-based medical record review study. Epidemiology. 2011;22:836-44.

9. Babikian T, Merkley T, Savage RC, Giza CC, Levin H. Chronic aspects of pediatric traumatic brain injury. Review of the Literature. J Neurotrauma. 2015. doi:10.1089/neu.2015.3971. Review of current state of knowledge about the chronic aspects of medical, cognitive, academic, emotional, behavioral, and family and social outcomes following pediatric TBI.

10. Babikian T, Asarnow R. Neurocognitive outcomes and recovery after pediatric TBI: meta-analytic review of the literature. Neuropsychology. 2009;23:283-96.

11. Anderson V, Catroppa C, Morse S, Haritou F, Rosenfeld J. Functional plasticity or vulnerability after early brain injury? Pediatrics. 2005;116:1374-82. Prosective longitudinal study plotting cognitive abilities after childhood TBI.

12. Ryan NP et al. Longitudinal outcome and recovery of social problems after pediatric traumatic brain injury (TBI): contribution of brain insult and family environment. Int J Dev Neurosci. 2016;49: 23-30.

13. Bigler ED. Anterior and middle cranial fossa in traumatic brain injury: relevant neuroanatomy and neuropathology in the study of neuropsychological outcome. Neuropsychology. 2007;21:515-31.

14.•- Tosetti $\mathrm{P}$ et al. Toward an international initiative for traumatic brain injury research. J Neurotrauma. 2013;30:1211-22. Summary document of a jointly sponsored workshop from The European Commission (EC) and the National Institutes of Health (NIH) organized to discuss the feasibility and benefits of an international collaboration in the field of traumatic brain injury (TBI) research.

15. Hyder AA, Wunderlich CA, Puvanachandra P, Gururaj G, Kobusingye OC. The impact of traumatic brain injuries: a global perspective. NeuroRehabilitation. 2007;22:341-53.

16. Roozenbeek B, Maas AIR, Menon DK. Changing patterns in the epidemiology of traumatic brain injury. Nat Rev Neurol. 2013;9: 231-6.
17. Marin JR, Weaver MD, Yealy DM, Mannix RC. Trends in visits for traumatic brain injury to emergency departments in the United States. JAMA. 2014;311:1917-9.

18. Zonfrillo MR et al. Prevalence of and risk factors for poor functioning after isolated mild traumatic brain injury in children. $\mathrm{J}$ Neurotrauma. 2014;31:722-7.

19. Faul M, Wald MM, Rutland-Brown W, Sullivent EE, Sattin RW. Using a cost-benefit analysis to estimate outcomes of a clinical treatment guideline: testing the Brain Trauma Foundation guidelines for the treatment of severe traumatic brain injury. J Trauma. 2007;63:1271-8.

20. Stein SC, Georgoff P, Meghan S, Mizra K, Sonnad SS. 150 years of treating severe traumatic brain injury: a systematic review of progress in mortality. J Neurotrauma. 2010;27:1343-53.

21. Clifton GL et al. Lack of effect of induction of hypothermia after acute brain injury. N Engl J Med. 2001;344:556-63.

22. Clifton GL et al. Very early hypothermia induction in patients with severe brain injury (the National Acute Brain Injury study: hypothermia II): a randomised trial. Lancet Neurol. 2011;10:131-9.

23. Hutchison JS et al. Hypothermia therapy after traumatic brain injury in children. N Engl J Med. 2008;358:2447-56. Pediatric trial of moderate hypothermia for the treatment of severe TBI in children.

24. Adelson PD et al. Comparison of hypothermia and normothermia after severe traumatic brain injury in children (Cool Kids): a phase 3, randomised controlled trial. Lancet Neurol. 2013;12:546-53. Pediatric trial of moderate hypothermia for the treatment of severe TBI in children.

25. Beca $\mathrm{J}$ et al. Hypothermia for traumatic brain injury in children - $\mathrm{a}$ phase ii randomized controlled trial. Crit Care Med. 2015;43:145866.

26. Skolnick BE et al. A clinical trial of progesterone for severe traumatic brain injury. N Engl J Med. 2014;371:2467-76.

27. Wright DW et al. Very early administration of progesterone for acute traumatic brain injury. N Engl J Med. 2014;371:2457-66.

28. Chesnut RM et al. A trial of intracranial-pressure monitoring in traumatic brain injury. N Engl J Med. 2012;367:2471-81. A prospective randomized-controlled trial of the use of ICP monitoring in patients with sTBI.

29. Chesnut RM. A conceptual approach to managing severe traumatic brain injury in a time of uncertainty. Ann N Y Acad Sci. 2015;1345: 99-107.

30. Wijayatilake DS, Talati C, Panchatsharam S. The monitoring and management of severe traumatic brain injury in the United Kingdom: is there a consensus?: a national survey. J Neurosurg Anesthesiol. 2015;27:241-5.

31. Piper LC et al. Guidelines for the treatment of severe traumatic brain injury: are they used? JAMA Surg. 2015;150:1013-5.

32. Shafi $\mathrm{S}$ et al. Suboptimal compliance with evidence-based guidelines in patients with traumatic brain injuries. J Neurosurg. 2014;120:773-7.

33. Lee JC et al. An analysis of Brain Trauma Foundation traumatic brain injury guideline compliance and patient outcome. Injury. 2015;46:854-8.

34. Hesdorffer DC, Ghajar J. Marked improvement in adherence to traumatic brain injury guidelines in United States trauma centers. J Trauma. 2007;63:841-7. discussion 847-8.

35. Fakhry SM, Trask AL, Waller MA, Watts DD. IRTC Neurotrauma Task Force. Management of brain-injured patients by an evidencebased medicine protocol improves outcomes and decreases hospital charges. The Journal of Trauma: Injury, Infection, and Critical Care. 2004;56:492-9. discussion 499-500.

36. Arabi YM et al. Mortality reduction after implementing a clinical practice guidelines-based management protocol for severe traumatic brain injury. J Crit Care. 2010;25:190-5. 
37. Dawes AJ et al. Compliance with evidence-based guidelines and interhospital variation in mortality for patients with severe traumatic brain injurY. JAMA Surg. 2015;150:965-72.

38. Bennett KS. Adherence to the guidelines for management of severe pediatric traumatic brain injury: enough evidence to support benefit?*. Crit Care Med. 2014;42:2308-9.

39. Alkhoury F, Kyriakides TC. Intracranial pressure monitoring in children with severe traumatic brain injury: national trauma data bank-based review of outcomes. JAMA Surg. 2014;149:544-8.

40. Keenan HT, Nocera M, Bratton SL. Frequency of intracranial pressure monitoring in infants and young toddlers with traumatic brain injury. Pediatr Crit Care Med. 2005;6:537-41.

41. Van Cleve $W$ et al. National variability in intracranial pressure monitoring and craniotomy for children with moderate to severe traumatic brain injury. Neurosurgery. 2013;73:746-52. discussion 752 quiz 752.

42. Roumeliotis N, Pettersen G, Crevier L, Emeriaud G. ICP monitoring in children: why are we not adhering to guidelines? Childs Nerv Syst. 2015. doi:10.1007/s00381-015-2837-9.

43. Morris KP et al. Intracranial pressure complicating severe traumatic brain injury in children: monitoring and management. Intensive Care Med. 2006;32:1606-12.

44. Brolliar SM et al. A qualitative study exploring factors associated with provider adherence to severe pediatric traumatic brain injury guidelines. J Neurotrauma. 2016. doi:10.1089/neu.2015.4183.

45. Vavilala MS et al. Acute care clinical indicators associated with discharge outcomes in children with severe traumatic brain injury. Crit Care Med. 2014;42:2258-66.

46. O'Lynnger TM et al. Standardizing ICU management of pediatric traumatic brain injury is associated with improved outcomes at discharge. J Neurosurg Pediatr. 2015;1-8. doi:10.3171/2015.5.PEDS1544.

47. Pineda JA et al. Effect of implementation of a paediatric neurocritical care programme on outcomes after severe traumatic brain injury: a retrospective cohort study. Lancet Neurol. 2013;12:45-52.

48. Zebrack $\mathrm{M}$ et al. Early resuscitation of children with moderate-tosevere traumatic brain injury. PEDIATRICS. 2009;124:56-64.

49. Lingsma HF et al. Between-centre differences and treatment effects in randomized controlled trials: a case study in traumatic brain injury. Trials. 2011;12:201.

50. Lingsma HF et al. Large between-center differences in outcome after moderate and severe traumatic brain injury in the international mission on prognosis and clinical trial design in traumatic brain injury (IMPACT) study. Neurosurgery 2011;68:601-7. discussion 607-8. Meta-analysis using random-effects logistic regression models to estimate the between-center differences in unfavorable outcome (dead, vegetative state, or severe disability measured with the Glasgow Outcome Scale) at 6 months for adult patients with sTBI.

51. Greene NH, Kernic MA, Vavilala MS, Rivara FP. Variation in pediatric traumatic brain injury outcomes in the United States. Arch Phys Med Rehabil. 2014;95:1148-55.

52. Mills B, Rowhani-Rahbar A, Simonetti JA, Vavilala MS. Facility characteristics and inhospital pediatric mortality after severe traumatic brain injury. J Neurotrauma. 2015;32:841-6.

53. Losonczy LI et al. The severity of disparity: increasing injury intensity accentuates disparate outcomes following trauma. J Health Care Poor Underserved. 2014;25:308-20.

54. Zonfrillo MR et al. Socioeconomic status and hospitalization costs for children with brain and spinal cord injury. J Pediatr. 2016;169: $250-5$.

55. Bell MJ et al. Differences in medical therapy goals for children with severe traumatic brain injury-an international study. Pediatr Crit Care Med. 2013;14:811-8.

56. Cremer OL et al. Effect of intracranial pressure monitoring and targeted intensive care on functional outcome after severe head injury. Crit Care Med. 2005;33:2207-13.
57. Shafi S, Diaz-Arrastia R, Madden C, Gentilello L. Intracranial pressure monitoring in brain-injured patients is associated with worsening of survival. J Trauma. 2008;64:335-40.

58. Duhaime AC, Rindler R. Special considerations in infants and children. In: Grafman J, Salazar AM, editors. Handbook of clinical neurology, traumatic brain injury, Part I, Elsevier: Philadelphia; 2015. p. 219-42.

59. Zhong W, Sima X, Huang S, Chen H, Cai B, Sun G, et al. Traumatic extradural hematoma in childhood. Childs Nerv Syst. 2013;29: 635-41.

60. Lahat E, Livne M, Barr J, Schiffer J, Eshel G. The management of epidural haematomas - surgical versus conservative management. Eur J Pediatrc. 1998;153:198-201.

61. Bell MJ, Kochanek PM. Pediatric traumatic brain injury in 2012 the year with new guidelines and common data elements. Crit Care Clin. 2012.

62. Adamo MA, Drazin D, Waldman JB. Decompressive craniectomy and postoperative complication management in infants and toddlers with severe traumatic brain injuries. J Neurosurg Pediatr. 2009;3(4):334.

63. Suarez EP, Gonzalez AS, Diaz CP, Salido AG, de Azagra Garde AM, Flores JC. Decompressive craniectomy in 14 children with severe head injury: clinical results with long-term follow-up and review of the literature. J Trauma - Injury Infection \& Critical Care. 2011;71(1):133-40.

64. Jagannathan J, Okonkwo DO, Dumont AS, Ahmed H, Bahari A, Prevedello DM et al. Outcome following decompressive craniectomy in children with severe traumatic brain injury: a 10year single-center experience with long-term follow up. J Neurosurgery, 2007. (4 Suppl) (268-75).

65. Oluigbo CO, Wilkinson CC, Stence NV, Fenton LZ, McNatt SA, Handler MH. Comparison of outcomes following decompressive craniectomy in children with accidental and nonaccidental blunt cranial trauma. J Neurosurg Pediatr. 2012;9(2):125.

66. Rocque BG, Amancherla K, Lew SM, Lam S. Outcomes of cranioplasty following decompressive craniectomy in the pediatric population. A systematic review. JNS Pediatrics. 2013;12:120-5.

67. Carney N, Kochanek P, Adelson D, Peterson K, Carson S, Ghajar J. Guidelines for the acute medical management of severe traumatic brain injury in infants, children, and adolescents, 2nd edition. Brain Inj. 2012;26(4-5):416-7.

67. Brenner DJ, Elliston CD, Hall EJ, Berdon WE. Estimated risks of radiation-induced fatal cancer from pediatric CT. AJR. 2001;176: 289-96. Studied calculation of organ doses of radiation as a function of age-at-diagnosis and estimated attributable lifetime cancer mortality risks (per unit dose) for different organ sites determined.

69. Brenner DJ, Hall EJ. Computed tomography — an increasing source of radiation exposure. N Engl J Med. 2007;357(22):2277-84.

70. Hall P, Adami H, Trichopoulos D, Pedersen N, Lagiou P, Ekbom A et al. Effect of low doses of ionising radiation in infancy on cognitive function in adulthood: Swedish population based cohort study. Br Med J. 2004;328(3 Jan 2004):19.

71. Goske MJ, Applegate KE, Bell C, Boylan J, Bulas D, Butler P, et al. Image Gently: providing practical educational tools and advocacy to accelerate radiation protection for children worldwide. Semin Ultrasound CT MR. 2010;31(1):57-63.

72. Cohen AR, Caruso P, Duhaime A-C, Klig JE. Feasibility of "rapid" magnetic resonance imaging in pediatric acute head injury. Am J Emerg Med. 2015;33(7):887-90.

73. Duhaime AC, Gean AD, Haacke EM, Hicks R, Wintermark M, Mukherjee $\mathrm{P}$, et al. Common data elements in radiologic imaging of traumatic brain injury. Arch Phys Med Rehabil. 2010;91(11): 1661-6.

74. Duhaime AC, Holshouser B, Hunter JV, Tong K. Common data elements for neuroimaging of traumatic brain injury: pediatric considerations. J Neurotrauma. 2012;29(4):629-33. 